\title{
RESEARCH
}

Open Access

\section{Glial activation is moderated by sex in response to amyloidosis but not to tau pathology in mouse models of neurodegenerative diseases}

Gloria Biechele ${ }^{1}$, Nicolai Franzmeier ${ }^{2}$, Tanja Blume ${ }^{1,3}$, Michael Ewers ${ }^{2,3}$, Jose Medina Luque ${ }^{3}$, Florian Eckenweber ${ }^{1}$, Christian Sacher ${ }^{1}$, Leonie Beyer ${ }^{1}$, Francois Ruch-Rubinstein ${ }^{1}$, Simon Lindner ${ }^{1}$, Franz-Josef Gildehaus ${ }^{1}$,

Barbara von Ungern-Sternberg ${ }^{1}$, Paul Cumming ${ }^{4,5}$, Peter Bartenstein ${ }^{1,7}$, Axel Rominger ${ }^{1,4}$, Günter U. Höglinger ${ }^{2,8,9}$, Jochen Herms $s^{2,7,6}$ and Matthias Brendel ${ }^{1,7^{*}}$ (D)

\begin{abstract}
Background: In vivo assessment of neuroinflammation by 18-kDa translocator protein positron-emission-tomography (TSPO-PET) ligands receives growing interest in preclinical and clinical research of neurodegenerative disorders. Higher TSPOPET binding as a surrogate for microglial activation in females has been reported for cognitively normal humans, but such effects have not yet been evaluated in rodent models of neurodegeneration and their controls. Thus, we aimed to investigate the impact of sex on microglial activation in amyloid and tau mouse models and wild-type controls.

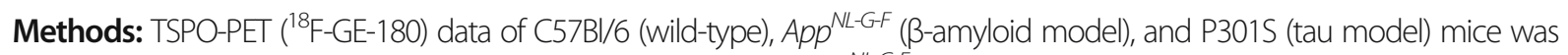
assessed longitudinally between 2 and 12 months of age. The $A p p^{N L-G F}$ group also underwent longitudinal $\beta$-amyloid-PET imaging (Aß-PET; ${ }^{18}$ F-florbetaben). PET results were confirmed and validated by immunohistochemical investigation of microglial (Iba-1, CD68), astrocytic (GFAP), and tau (AT8) markers. Findings in cerebral cortex were compared by sex using linear mixed models for PET data and analysis of variance for immunohistochemistry.

Results: Wild-type mice showed an increased TSPO-PET signal over time (female $+23 \%$, male $+4 \%$ ), with a significant sex $\times$ age interaction $(T=-4.171, p<0.001)$. The $A \beta$ model $A p p^{N L-G-F}$ mice also showed a significant sex $\times$ age interaction $\left(T=-2.953, p=0.0048\right.$ ), where cortical TSPO-PET values increased by $31 \%$ in female $A p p^{N L-G-F}$ mice, versus only $6 \%$ in the male mice group from 2.5 to 10 months of age. Immunohistochemistry for the microglial markers Iba- 1 and CD68 confirmed the TSPO-PET findings in male and female mice aged 10 months. AB-PET in the same App ${ }^{N L-G-F}$ mice indicated no significant sex $\times$ age interaction $(T=0.425, p=0.673)$. The P301S tau model showed strong cortical increases of TSPO-PET from 2 to 8.5 months of age (female $+32 \%$, male $+36 \%$ ), without any significant sex $\times$ age interaction ( $T=-0.671, p=0.504)$, and no sex differences in lba-1, CD68, or AT8 immunohistochemistry.

\footnotetext{
* Correspondence: matthias.brendel@med.uni-muenchen.de

'Department of Nuclear Medicine, University Hospital of Munich, LMU

Munich, Marchioninstraße 15, 81377 Munich, Germany

${ }^{7}$ Department of Neurology, Hannover Medical School, Hannover, Germany

Full list of author information is available at the end of the article
}

(c) The Author(s). 2020 Open Access This article is licensed under a Creative Commons Attribution 4.0 International License, which permits use, sharing, adaptation, distribution and reproduction in any medium or format, as long as you give appropriate credit to the original author(s) and the source, provide a link to the Creative Commons licence, and indicate if changes were made. The images or other third party material in this article are included in the article's Creative Commons licence, unless indicated otherwise in a credit line to the material. If material is not included in the article's Creative Commons licence and your intended use is not permitted by statutory regulation or exceeds the permitted use, you will need to obtain permission directly from the copyright holder. To view a copy of this licence, visit http://creativecommons.org/licenses/by/4.0/ The Creative Commons Public Domain Dedication waiver (http://creativecommons.org/publicdomain/zero/1.0/) applies to the data made available in this article, unless otherwise stated in a credit line to the data. 
(Continued from previous page)

Conclusion: Female mice indicate sex-dependent microglia activation in aging and in response to amyloidosis but not in response to tau pathology. This calls for consideration of sex difference in TSPO-PET studies of microglial activation in mouse models of neurodegeneration and by extension in human studies.

Keywords: Sex, Microglia, TSPO, Amyloid, Tau

\section{Introduction}

Alzheimer's disease (AD) is the most prevalent neurodegenerative disease in societies with aging populations [1]. The neuropathology of AD is characterized by the histological triad of accumulation of extracellular amyloid- $\beta$ peptide $(A \beta)$ plaques, fibrillary tau aggregates within neurons, and the activation of neuroinflammatory pathways mediated by microglia and astrocytes [2-6]. Importantly, the assessment of glial activation by $18-\mathrm{kDa}$ translocator protein (TSPO) positron emission tomography (TSPO-PET) has received growing interest in the last decade [7], which has spurned the development of a wide range of tracers for human studies [8]. Molecular imaging for stratification and monitoring of glial activation could become crucial for target engagement and response assessment of immunomodulatory therapies [9].

Human clinical data indicate that men and women exhibit sex differences in the neuropathological and symptomatic progression of AD [10]. The lifetime risk of later developing $\mathrm{AD}$ for 65-year-old females is twice that of men of the same age (12\% vs. $6.3 \%$, respectively) [11]. Women also show faster progression from mild cognitive impairment to $\mathrm{AD}$ dementia when compared to men [12]. There is growing evidence that sex differences in neuroinflammation pathways including microglia could play a crucial role in driving the sex differences observed in $\mathrm{AD}$ [13]. Nonetheless, the few reports on sex differences in TSPO expression have mainly focused on astrocytes in culture. For example, cultured astrocytes derived from female mouse pups showed stronger increases in TSPO mRNA expression upon exposure to bacterial lipopolysaccharide challenge that did cultured astrocytes derived from male littermates [14]. Notably, glial cells express receptors for estrogens and androgens, suggesting the potential for modulation of neuroinflammatory responses by sex steroid hormones [15-17]. Furthermore, a key function attributed to TSPO in microglia is cholesterol transport within the mitochondria [18], which implies downstream effects on the synthesis and metabolism of sex steroid hormones.

A recent human study in cognitively healthy individuals revealed significant sex differences of ${ }^{11}$ C-PBR28 binding in brain, with women showing a higher TSPOPET signal [17]. However, although TSPO-PET is increasingly used for investigation in vivo of microglial activation in mouse models of AD [19-22], preclinical studies have not previously considered possible sex effects on TSPO-PET findings.

Given this background, we aimed to investigate sex differences in the TSPO-PET signal in wild-type mice as well as in mouse models of $A \beta$ and tau pathology. We tested if the observed effects are independent from potential sex differences in protein aggregation in these models. Finally, we aimed to elucidate if our effects are specific to TSPO-PET binding or if they can be validated by immunostaining for microglia.

\section{Material and methods \\ Animals and study design}

All experiments were performed in compliance with the National Guidelines for Animal Protection, Germany, and with the approval of the regional animal committee (Regierung Oberbayern), with oversight by a veterinarian. Mice were housed in a temperature- and humiditycontrolled environment with a 12-h light-dark cycle and with free access to food (Sniff, Soest, Germany) and water. Males and females were housed separately in the same animal facility, with cages of equal size and comparable handling. A larger sample of female mice was a priori included in the longitudinal studies listed below due to the higher frequency of aggressive behavior among male cage mates, which can cause space issues when they need to be separated. Cage mates were kept together in small cohorts when entering the imaging facility and no mice had to be separated during longitudinal imaging. All mice with longitudinal imaging data were included in the analysis as stated below. A detailed overview on the sample size of the mouse cohort is provided in Table 1.

All PET raw data is derived previous in-house studies conducted using the same tomograph and with identical acquisition parameters. The raw data were reprocessed to obtain maximal agreement of spatial and activity normalization between studies. We included data from descriptive datasets or control groups of therapy/genotype studies:

- C57BL/6: Historical cross-sectional ${ }^{18} \mathrm{~F}-\mathrm{GE}-180$ data from 126 scans of wild-type C57BL/6 mice aged 213 months were reanalyzed. Iba- 1 staining was performed in four wild-type mice of each sex at 12-14 months of age. In total, 86 of the 126 (68\%) TSPO- 
Table 1 Sample sizes of the mouse cohorts across PET and histo- and immunohistochemistry modalities

\begin{tabular}{|c|c|c|c|c|c|c|c|c|c|c|c|}
\hline \multirow{2}{*}{$\begin{array}{l}\text { Genotype } \\
\text { Sex }\end{array}$} & & \multicolumn{2}{|l|}{$2-3 M$} & \multicolumn{2}{|c|}{$4-5 M$} & \multicolumn{2}{|c|}{ 6-7 M } & \multicolumn{2}{|c|}{ 8-11 M } & \multicolumn{2}{|c|}{$12-14 M$} \\
\hline & & Male & Female & Male & Female & Male & Female & Male & Female & Male & Female \\
\hline \multirow[t]{2}{*}{ C57BL/6 } & TSPO-PET & 16 & 31 & 9 & 18 & 2 & 5 & 5 & 27 & 4 & 9 \\
\hline & |ba-1 & & & & & & & & & 4 & 4 \\
\hline \multirow[t]{6}{*}{$A p p^{N L-G-F}$} & TSPO-PET & 6 & 6 & 6 & 15 & 6 & 15 & 6 & 15 & & \\
\hline & $A \beta-P E T$ & 6 & 6 & 6 & 15 & 6 & 15 & 6 & 15 & & \\
\hline & Iba-1 & & & & & & & 4 & 5 & & \\
\hline & CD68 & & & & & & & 4 & 4 & & \\
\hline & Methoxy-x04 & & & & & & & 4 & 5 & & \\
\hline & GFAP & & & & & & & 4 & 4 & & \\
\hline \multirow[t]{4}{*}{ P301S } & TSPO-PET & 19 & 33 & 18 & 32 & 15 & 27 & 6 & 6 & & \\
\hline & Iba-1 & & & & & & & 7 & 7 & & \\
\hline & CD68 & & & & & & & 7 & 7 & & \\
\hline & AT8 & & & & & & & 7 & 7 & & \\
\hline
\end{tabular}

PET scans of C57BL/6 mice were imaged in parallel with $A p p^{N L-G-F}$ and P301S mouse models, whereas the remaining 40 mice (32\%) were imaged within the same time-period (2016-2020) but not in parallel with $A p p^{N L-G-F}$ and P301S mice included in the current study.

- $A p p^{N L-G-F}\left(A p p^{N L-G-F / N L-G-F}\right)$ : The knock-in mouse model $A p p^{N L-G-F}$ carries a mutant amyloid precursor protein (APP) gene encoding the humanized A $\beta$ sequence (G601R, F606Y, and R609H) with three pathogenic mutations, namely Swedish (KM595/ 596NL), Beyreuther/Iberian (I641F), and Arctic (E618G). Homozygotic $A p p^{N L-G-F}$ mice progressively exhibit widespread cerebral $A \beta$ accumulation from 2 months of age $[23,24]$. Longitudinal ${ }^{18} \mathrm{~F}-\mathrm{GE}-180$ data from 75 serial scans of homozygotic $A p p^{N L-G-F}$ mice imaged at four different ages $(2.5,5.0,7.5$, and 10 months) from previous $[25,26]$ and unpublished studies were reprocessed. Six mice of each sex were imaged longitudinally at all four time-points whereas nine females entered PET imaging starting from 5.0 months of age. Only mice with successful imaging until 10 months of age were included. The average cage occupancy ( $n$ per cage) was 2.1 for females and 1.5 for males. All these mice had a contemporaneous ${ }^{18}$ F-florbetaben A $\beta$-PET scan, which was reprocessed and analyzed analogously. Iba-1, CD68, GFAP, and methoxy-X04 staining in four to five $A p p^{N L-G-F}$ mice of each sex at 11 months of age.

- P301S: P301S mice express the human 0N4R tau isoform with the P301S mutation in exon 10 of MAPT gene, which is under control of the murine thy1 promoter on a C57BL/6 background [27, 28]. This model develops aggregates of hyperphosphorylated tau mainly in the brainstem, with onset starting at 2-3 months of age. Tau filaments in these mice appear mostly as half-twisted ribbons, with a lesser abundance of larger paired helical tau filaments resembling those seen in human AD patients. The behavioral phenotype of P301S mice manifests as learning deficits from 2-3 months of age, and onset of motor impairment at 4 months, leading to early death before 12 months of age. Longitudinal ${ }^{18}$ F-GE-180 data from 156 serial scans of P301S mice imaged at up to four different timepoints (2, 4, 6, and 8 months) from a previous [21] and an unpublished study were reprocessed. Six mice of each sex were imaged longitudinally at all four time-points whereas 27 female and 13 male mice were imaged from 2 to 6 months of age. Failed imaging sessions at follow-up time-points were excluded. The average cage occupancy ( $n$ per cage) was 2.6 for females and 1.9 for males. Iba-1, CD68, and AT8 staining had been performed in seven homozygotic P301S mice of each sex at 7-8 months of age.

\section{PET imaging}

PET data acquisition, reconstruction, and post-processing

For all PET procedures, including radiochemistry, acquisition and pre-processing, we used an established and standardized protocol [19, 29]. In brief, ${ }^{18}$ F-GE-180 TSPO-PET recordings (average dose $12.3 \pm 2.2 \mathrm{MBq}$ ) with an emission window of 60-90 min after injection were obtained to measure glial activation. ${ }^{18} \mathrm{~F}$-florbetaben $\mathrm{A} \beta$-PET recordings (average dose $11.8 \pm 1.9 \mathrm{MBq}$ ) with an emission window of 30-60 min after injection were performed for assessment of fibrillar $A \beta$ accumulation. Anesthesia was induced with $3.0 \%$ isoflurane delivered via a mask at $3.5 \mathrm{~L} / \mathrm{min}$, with maintenance of 
anesthesia by $1.5 \%$ isoflurane during the imaging time window.

\section{PET image analysis}

We performed all PET data analyses using PMOD (version 3.5; PMOD technologies). Normalization of TSPOPET emission images was performed by standardized uptake value ratios (SUVR) using previously established reference tissues in the WT (white matter), $A p p^{N L-G-F}$ (periaqueductal grey), and P301S (nucleus accumbens) mice $[19,25,29,30]$. Two bilateral volumes of interest placed in the frontal cortex (comprising $15 \mathrm{~mm}^{3}$ each) served for calculation of target-to-reference SUVR. We chose frontal cortex for quantification of TSPO-PET signal based on earlier findings in C57BL/6, $A p p^{N L-G-F}$, and P301S mice [21, 25, 30], and due to the documented presence of neuropathology in $A p p^{N L-G-F}$ and P301S mice, along with the requirement for a sufficiently large standard volume of interest (VOI).

\section{Immunohistochemistry}

Iba-1, CD68, methoxy-X04, and AT8 immuno- and histochemical stainings were performed as described previously $[21,25]$. In brief, we performed a standard free-floating immunofluorescence protocol cortex and brainstem areas matching the PET brain regions. As previously described, perfusion fixed $50-\mu \mathrm{m}$-thick brain sections were rinsed either overnight or for $48 \mathrm{~h}$ in PBS with $0.2 \%$ Triton X-100 containing one of the following primary antibodies: rabbit monoclonal Iba-1 (1:500. Wako: 19-19741), rat monoclonal CD68 (1:500. Bio-Rad: MCA1857), and mouse monoclonal phospho-Tau (1: 500. Thermo Fisher: MN1020). After washing in PBS, sections were then incubated in a combination of three secondary antibodies (Alexa 488 goat anti-rabbit, Alexa 594 goat anti-mouse, and Alexa 647 goat anti-rat IgG). Following this step, $A \beta$ fibrils were stained for $25 \mathrm{~min}$ upon addition of methoxy-X04 $(10 \mu \mathrm{g} / \mathrm{mL}$ in $50 \%$ ethanol) at room temperature, followed by washing in buffer (4 times ten min each).

For GFAP staining, free-floating sections were blocked with Normal Goat serum (5\%) in PBS + 0.3\% Triton X100 for $1 \mathrm{~h}$ at room temperature. We obtained immunohistochemical labelling of astrocytes using the antibody anti-GFAP (Synaptic Systems) 1:500 in 1\% NGS blocking solution overnight at $4{ }^{\circ} \mathrm{C}$ and the Alexa Fluor 555 secondary antibody anti-rabbit (Invitrogen) 1:500 in 1\% NGS blocking solution for $3 \mathrm{~h}$ at room temperature. The unbound dye was removed by three washing steps with PBS, and the slices were then mounted on microscope slides with fluorescent mounting medium (Dako, Germany).

We performed quantitation in ImageJ (https://imagej. nih.gov/ij/) with images obtained from a confocal microscope (LSM 780 Axio invers). For each marker, we calculated the percentage coverage of positive staining in a cortical region matching the PET VOI.

\section{Statistics}

In wild-type mice, we first assessed whether there was an age-dependent increase in TSPO-PET signal, using a linear mixed model with age at scanning as a predictor of TSPO-PET controlling for sex (fixed effect) as well as for random slope and intercept. Next, we tested for sex differences in the age dependence of TSPO-PET results by additionally including a sex $\times$ age interaction as a predictor.

We applied equivalent models in the $A p p^{N L-G-F}$ mice both for TSPO-PET and A $\beta$-PET, and likewise in the P301S tau transgenic mice for TSPO-PET, to determine whether sex moderated the age-dependent PET increases. When assessing the sex by age interaction effect on the increases in TSPO-PET binding in the $A p p^{N L-G-F}$ mice, we additionally controlled the models for individual concomitant A $\beta$-PET levels to isolate sex-specific effects on the TSPO-PET increases from confound due to the primary fibrillar $\mathrm{A} \beta$ pathology.

Effects of sex on histo- and immunohistochemistry area-\% readouts were tested by unpaired Student's $t$ test, and effect sizes were calculated as Cohen's $d$. Statistical analyses were performed in $\mathrm{R}$ using the lme4 package for linear mixed models. For all models, we applied an alpha threshold of 0.05 for considering effects to be statistically significant.

\section{Results}

Stronger elevation of the cortical TSPO-PET signal in females is reproducible in rodents

Our first objective was to investigate if recent human findings of higher TSPO-PET signal in cognitively healthy females when compared to males [17] also hold true for healthy wild-type mice. Therefore, we availed ourselves of our large $\mu$ PET database to make a crosssectional comparison of ${ }^{18} \mathrm{~F}$-GE-180 TSPO-PET scans by sex. Wild-type mice showed increasing TSPO-PET SUVR in cortex with age $(T=8.915, \mathrm{~b} / \mathrm{SE}=0.009 / 0.001$, $p<0.001$, Cohen's $d=1.608$ ), in line with our earlier report on this phenomenon [30]. Furthermore, we found a significant age $\times$ sex interaction $(T=-4.171, \mathrm{~b} / \mathrm{SE}=-$ $0.009 / 0.002, p<0.001$, Cohen's $d=0.755)$ in these animals, controlling for slope (age) and random intercept, indicating that female mice showed more pronounced TSPO-PET SUVR increases than did male mice (Fig. 1a). Immunohistochemistry confirmed higher Iba-1 quantification in female wild-type mice at 12-14 months of age when compared to males $(3.23 \pm 0.09 \%$ vs. $2.97 \pm 0.10 \%$, $p=0.010$, Cohen's $d=1.87$; Fig. 2a). In summary, we confirmed our hypothesis that (as seen in cognitively intact humans) female wild-type mice would show greater 

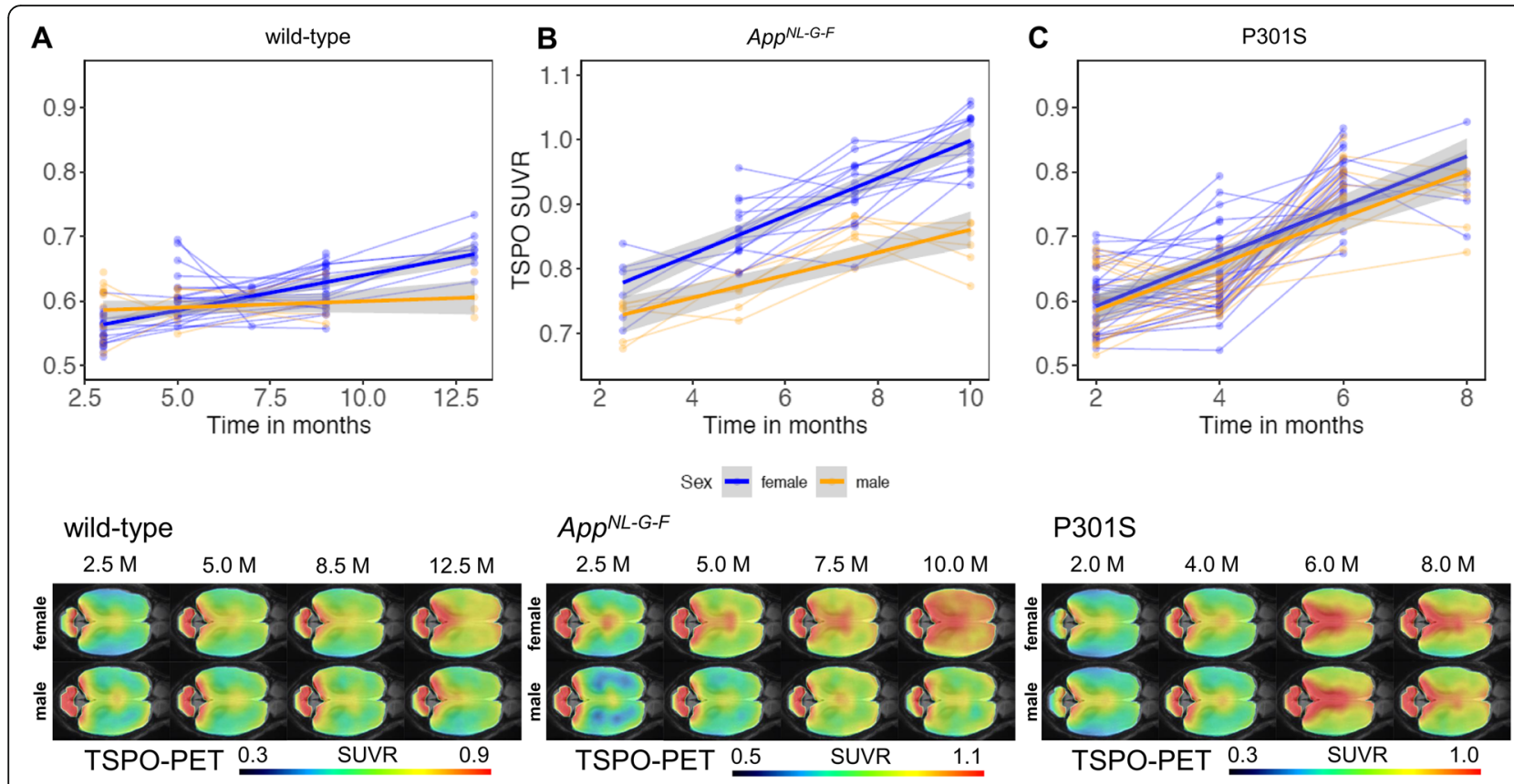

Fig. 1 Sex effects on TSPO-PET signal in wild-type mice and mouse models of neurodegenerative diseases. Cortical TSPO-PET quantification is illustrated as a function of age for female (blue) and male (yellow) mice, analyzed by mixed linear models, together with ${ }^{18}$ F-GE-180 TSPO-PET group mean images $(n=5-32)$ at different ages in a horizontal plane projected upon an MRI standard template. Serial scans in the upper part of the figure are indicated by connected data points. 95\% confidence intervals are indicated in grey. a Female wild-type mice showed a stronger increase of TSPO-PET SUVR than males $(p<0.001)$ from 2.5 to 12.5 months. b Female App $p^{N L-G-F}$ mice with A $\beta$ pathology already showed higher SUVR of TSPO-PET at 2.5 months and stronger increases of TSPO-PET SUVR from 2.5 to 10 months as compared to males $(p=0.0048)$. $\mathbf{c}$ P301S tau transgenic mice showed no significant sex $\times$ time interaction on TSPO-PET SUVR with age at time of scanning $(p=0.673)$. TSPO-translocator protein, PET—positron emission tomography, MRI—-magnetic resonance imaging, SUVR—standardized uptake value ratio, A —beta amyloid, M-months

age-dependent increases in TSPO-PET binding, which was evident by diverging slopes of female and male mice starting from 6 to 7 months of age.

\section{Sex-specific TSPO-PET increase in the presence of $A \beta$ pathology}

Next, we tested whether female $A p p^{N L-G-F}$ mice showed greater longitudinal increases in microglial activation, using TSPO-PET as a proxy. There was a pronounced TSPO-PET increase in the cortex of $A p p^{N L-G-F}$ mice with age when considering both female and male mice $(T=$ $11.831, \mathrm{~b} / \mathrm{SE}=0.024 / 0.002, p<0.0001$, Cohen's $d=5.893$, linear mixed model, adjusted for sex, random slope, and intercept). We then proceeded to test for a sex $\times$ age interaction for TSPO-PET SUVR, controlling for random slope (i.e., age at scanning) and intercept. As hypothesized, we found a significant sex $\times$ age interaction for TSPO-PET SUVR $(T=-2.953, \mathrm{~b} / \mathrm{SE}=-0.011 / 0.004, p=0.0048$, Cohen's $d=0.827$, Fig. $1 \mathrm{~b}$ ), where the female $A p p^{N L-G-F}$ mice showed a greater increase in TSPO-PET SUVR with age than did male mice.

To account for potential sex differences in fibrillar $A \beta$ aggregation, we tested whether $A p p^{N L-G-F}$ mice showed sex differences in A $\beta$-PET. Using longitudinal ${ }^{18} \mathrm{~F}$ -
florbetaben-PET data in the same mice, we tested the sex $\times$ age interaction on ${ }^{18} \mathrm{~F}$-florbetaben-PET SUVR, using linear mixed models controlling for random slope (i.e., age) and intercept. There was no significant sex $\times$ age interaction on ${ }^{18} \mathrm{~F}$-florbetaben-PET SUVRs $(T=$ $0.425, \mathrm{~b} / \mathrm{SE}=0.001 / 0.003, p=0.673$, Cohen's $d=0.141$, Fig. 3a). Thus, we conclude that the longitudinal increases of fibrillar $A \beta$ aggregation in cortex are comparable between male and female $A p p^{N L-G-F}$ mice (main effect of age on ${ }^{18} \mathrm{~F}$-florbetaben-PET SUVR, controlling for sex, random slope, and intercept: $T=6.048, \mathrm{~b} / \mathrm{SE}=$ $0.009 / 0.002, p<0.0001$, Cohen's $d=1.872$ ). More importantly, the aforementioned sex effect on TSPO-PET remained consistent when controlling for ${ }^{18} \mathrm{~F}$-florbetaben-PET SUVR at each time-point $(T=-2.926, \mathrm{~b} / \mathrm{SE}=$ $-0.011 / 0.0038, p=0.0051$, Cohen's $d=0.820$ ), suggesting that sex differences in TSPO-PET SUVR are not driven by sex-specific differences in fibrillar $A \beta$ burden. Immunohistochemistry at the terminal time-point confirmed the observations in vivo, showing higher expression of activated microglial markers in the female mice compared to males (Iba-1: $11.14 \pm 1.02 \%$ vs. $7.44 \pm$ $0.91 \%, p=0.0008$, Cohen's $d=2.71$; CD68: $3.01 \pm 0.06 \%$ vs. $1.57 \pm 0.13 \%, p=1 \mathrm{E}-6$, Cohen's $d=10.13$ ) (Fig. 2b). 


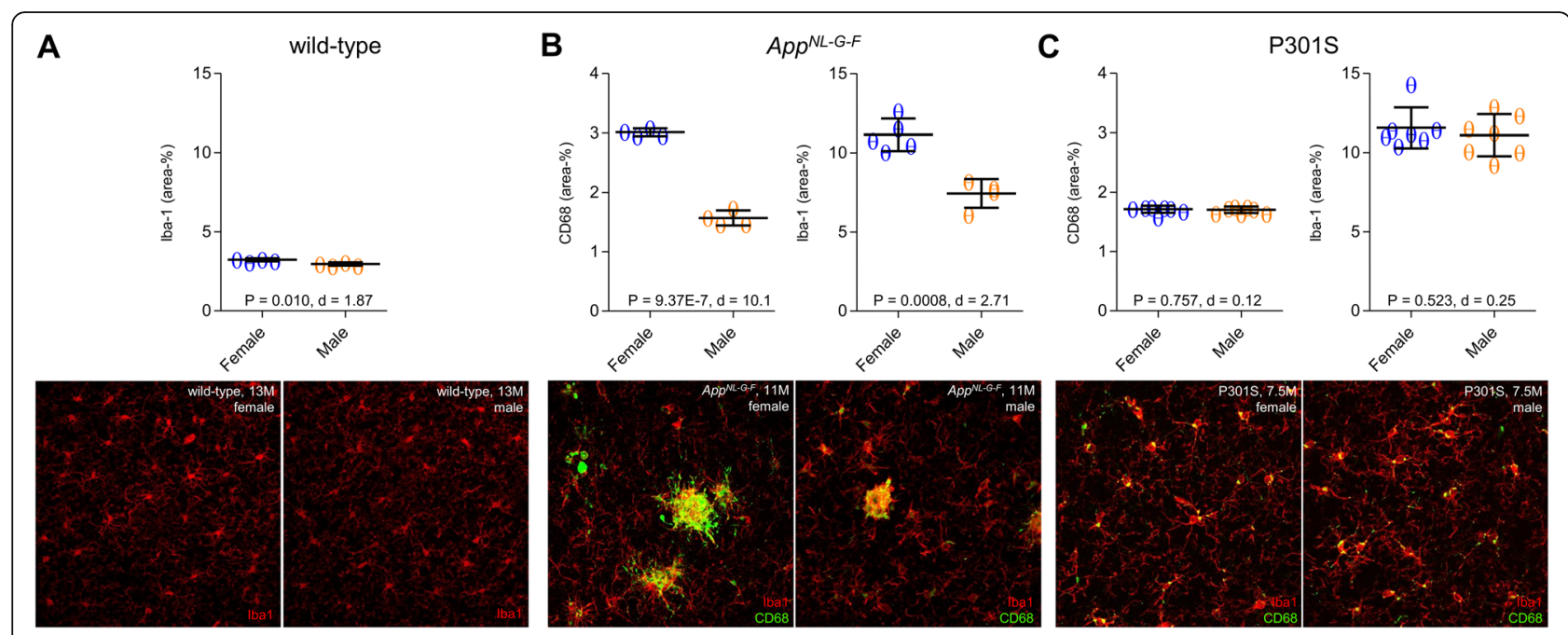

Fig. 2 Immunohistochemistry validation. Scatter plots and representative images of Iba-1 and CD68 immunostaining in male and female a wildtype, b App $p^{N L-G-F}$, and c P301S mice

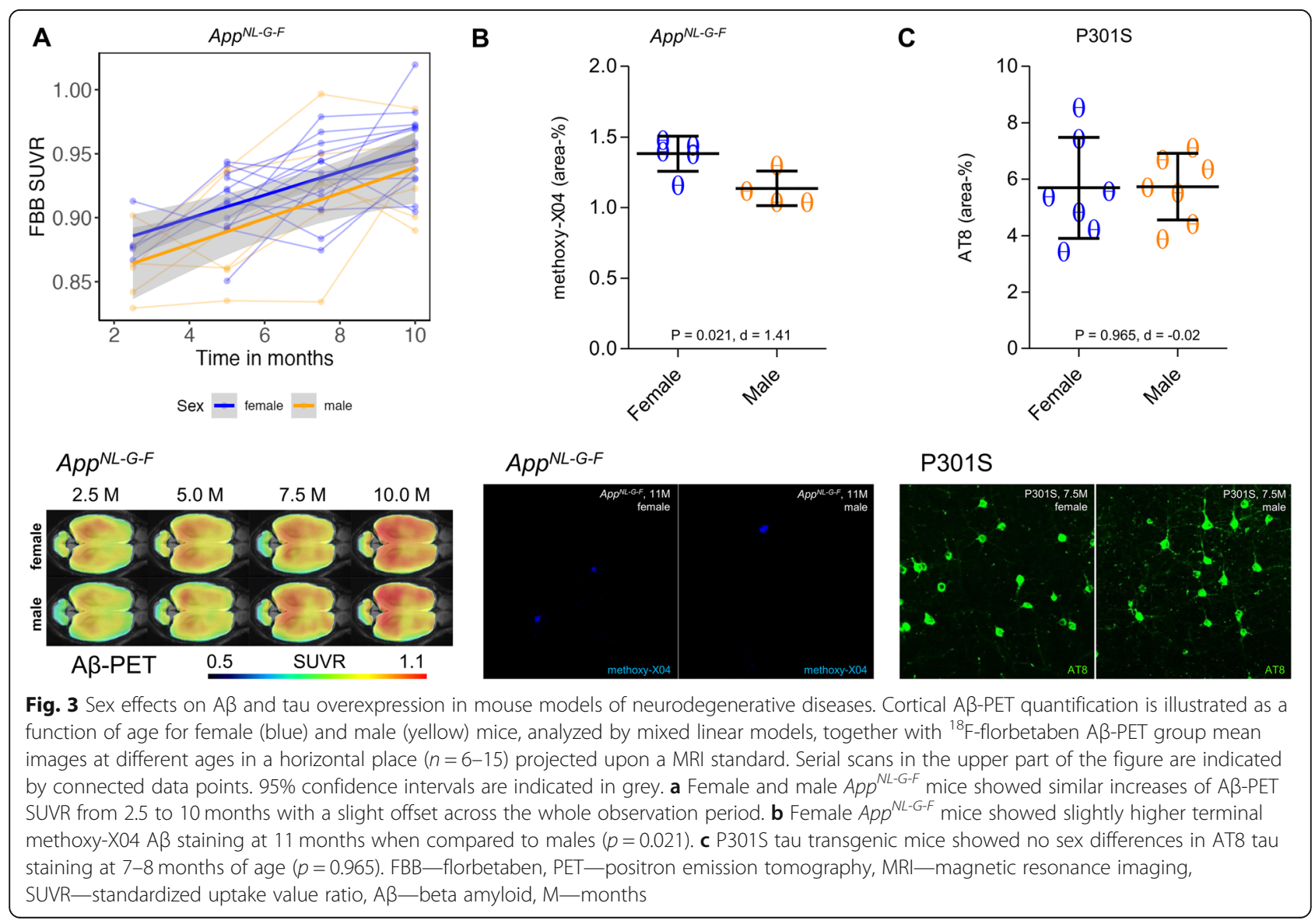


Methoxy-X04 histology indicated slightly higher fibrillar A $\beta$ levels in $A p p^{N L-G-F}$ females at the terminal timepoint $(1.38 \pm 0.12 \%$ vs. $1.14 \pm 0.12 \%, p=0.021$, Cohen's $d=1.41$, Fig. 3b). GFAP immunohistochemistry showed slightly higher levels of reactive astrocytes in $A p p^{N L-G-F}$ females at the terminal time-point $(7.23 \pm 1.00 \%$ vs. 5.60 $\pm 0.28 \%, p=0.020$, Cohen's $d=1.58$, Fig. 4).

In summary, we find a striking effect of sex on the age-dependent increase in cortical TSPO-PET signal in $\mathrm{A} \beta$-transgenic mice, in the absence of notable sex differences in fibrillar $A \beta$ pathology to ${ }^{18} \mathrm{~F}$-florbetaben-PET SUVR, and with only slightly higher fibrillar $A \beta$ to histology at the terminal time-point. This indicates that the sex difference in microglial activation is not readily attributable to sex differences of fibrillar $A \beta$ pathology.

\section{TSPO-PET increases in response to tau pathology are not moderated by sex}

Next, we tested whether sex-specific increases of the TSPO-PET signal were specifically related to cerebral $A \beta$ accumulation, or rather a more general function of AD-like brain pathology. To this end, we assessed longitudinal TSPO-PET in P301S tau transgenic mice, finding the expected longitudinal increases in TSPO-PET SUVR with age $(T=15.77, \mathrm{~b} / \mathrm{SE}=0.038 / 0.002, p<0.0001$, Cohen's $d=2.987$ ). There was, however, no significant sex $\times$ age interaction with TSPO-PET SUVR, controlling for slope (i.e., age) and random intercept $(T=-0.671$, $\mathrm{b} / \mathrm{SE}=-0.003 / 0.005, p=0.504$, Cohen's $d=0.128$, Fig. 1c). Thus, the TSPO-PET SUVR increases in response to 4-repeat isoform tau pathology did not differ between male and female P301S mice. Terminal immunohistochemical analysis likewise did not indicate any significant sex differences in microglial markers (Iba-1, $11.56 \pm 1.29 \%$ vs. $11.10 \pm 1.33 \%, p=0.523$, Cohen's $d=$ 0.25 ; CD68, $1.72 \pm 0.06 \%$ vs. $1.71 \pm 0.05 \%, p=0.757$, Cohen's $d=0.12$; Fig. 2c) or AT8-positive tau accumulation $(5.69 \pm 1.79 \%$ vs. $5.73 \pm 1.18 \%, p=0.965$, Cohen's $d$ $=-0.02$; Fig. 3c) in P301S mice aged 7-8 months.

\section{Discussion}

This is the first investigation aiming to elucidate sex differences in microglial activation in aging wild-type mice and mouse models of AD-like pathologies. Our data show a sex-specific effect on the cortical TSPO-PET signal and immunohistochemical markers of microgliosis in wild-type mice, with a greater age-dependent increase in female mice, thus in line with human TSPO-PET data in cognitively healthy men and women. Notably, we also observed a strong sex-specific effect on TSPO-PET signal during accumulation of $\mathrm{A} \beta$ pathology, indicating higher TSPO-PET binding in female $A p p^{N L-G-F}$ mice than in males. Contemporaneous A $\beta$-PET from the same mice allowed us to adjust TSPO-PET results for possible confounding effects due to sex differences in the progression of fibrillar $A \beta$ pathology. In contrast, P301S mice did not indicate sex differences in TSPO-PET signal increases in response to their accumulation of tau pathology. Terminal histo- and immunohistochemical analyses of phosphorylated tau, $A \beta$, and microglial markers confirmed all of the main PET findings.

Similar to a recent multi-center TSPO-PET in cognitively healthy men and women [17], we observed a strong effect of sex on the cortical TSPO-PET signal in a cross-sectional cohort of wild-type mice, indicating progressively higher TSPO-PET signal in cortex of females as compared to males. Importantly, we used data from mice housed under standard conditions, and with a completely matched TSPO-PET protocol, thus minimizing variance from methodological and environmental factors. This is critically important, given our compilation of data from various studies.

The human TSPO-PET study showed a more distinctly higher grey matter signal in younger women, which declined with increasing age of the subjects [17]. In contrast, our wild-type mouse data showed a sex difference in TSPO-PET favoring the females, which became more prominent with increasing age. However, we note that our tracking of TSPO-PET extended to only to 13 months of age, which is perhaps comparable to

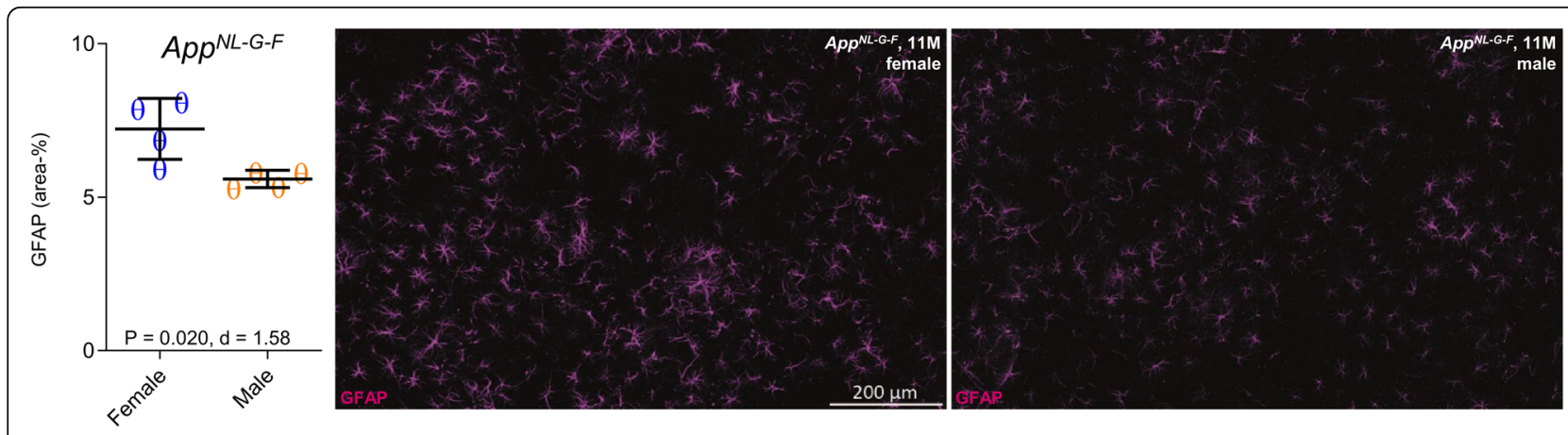

Fig. 4 Reactive astrocytes in $A p p^{N L-G-F}$ mice. Scatter plots and representative images of GFAP immunostaining in male and female App $p^{N L-G-F}$ mice 
middle age in humans [31]. Therefore, sex differences in TSPO-PET may follow differing time-courses in healthy mice and humans in relation to their vastly different lifespans. Our histo- and immunohistochemical validations of the in vivo TSPO-PET results present a distinct advantage of our preclinical study. Thus, we unambiguously establish the congruence of TSPO-PET findings with greater elevation of the microglia marker Iba-1 in aged female wild-type mice when compared to males. This result also supports the claims made in the human study cited above that increasing TSPO-PET binding with healthy aging is attributable to microglial activation, i.e., neuroinflammation. The large variability of human TSPO-PET binding in healthy individuals [17] and the allelic dependence of most TSPO tracers [8] predict a need for substantial group sizes to detect sex interactions with TSPO-PET signal in neurodegenerative diseases like AD. Our present use of inbred mouse lineages may have tended to reduce variance in the various endpoints, and our use of transgenic mouse models with overexpression of $A \beta$ or hyperphosphorylated tau allows the isolation of effects of different protein aggregates on the interaction between sex and TSPO-PET expression. While preclinical $\mathrm{AD}$ patients can show $\mathrm{A} \beta$-positivity and tau-negativity [32], there is lingering uncertainty about the threshold of disease progression for detection of tau in cerebrospinal fluid or tau-PET assessments [33]. However, our investigation reveals a sex-specific effect on TSPO-PET increases during accumulation of $\mathrm{A} \beta$ pathology in $A p p^{N L-G-F}$ mice, with an interaction of a magnitude similar to that seen in aging wild-type mice. The progression of $A \beta$ pathology is more rapid in female AD-model mice than in males [34]. A primary accumulation of fibrillar $A \beta$ triggers a secondary neuroinflammation mediated by activated microglia [35]. Preliminary results had indicated sex differences in $\mathrm{A} \beta$ accumulation in $A p p^{N L-G-F}$ mice, albeit with onset in the interval between 12 and 18 months [23], thus occurring after the present observation period. We did not observe sex differences of the A $\beta$-PET increase in $A p p^{N L-G-F}$ mice and inclusion of A $\beta$-PET results in the linear mixed model did not alter the sex difference in TSPO-PET. MethoxyX04 staining indicated moderately higher fibrillar $A \beta$ pathology in female $A p p^{N L-G-F}$ mice compared to male $A p p^{N L-G-F}$ mice, which may reflect greater sensitivity of the histological staining [20], or the preponderant weighting of the A $\beta$-PET signal to fibrillar $A \beta$ and its insensitivity to soluble $A \beta$ proportions. Thus, we cannot exclude the possibility that the effect of sex on TSPOPET in $A p p^{N L-G-F}$ mice may be influenced by sex differences in the biochemical nature of the $A \beta$ pathology. While the main attribution of the TSPO-PET signal is to activate microglia, there is also a contribution from proinflammatory astrocytes [36]. We note that the proportion of astroglial versus microglial contributions to TSPO-PET signals might differ between male and female mice and our immunohistochemistry data showed sex differences for both CD68 reactivity and GFAP reactivity in $A p p^{N L-G-F}$ mice. A direct correlation analyses between TSPO-PET and immunohistochemistry in larger cohorts of male and female $A p p^{N L-G-F}$ mice could better substantiate the cellular origin of the TSPO-PET signal.

Surprisingly, we were unable to detect corresponding sex-specific effects on longitudinal TSPO-PET increases in the P301S tau mouse model. Negative PET findings are supported by the absence of sex differences in Iba- 1 and CD68 immunohistochemistry, and by the equal levels of hyperphosphorylated AT8-positive tau in female and male P301S mice at 7 months of age. Although there were significant sex differences in microglial microRNA expression model mice [37], our present in vivo findings do not indicate any sex difference in the TSPO-PET binding with aging of P301S mice. Indeed, these tauopathy model mice did not manifest the sex effect seen in wild-type mice, perhaps due to masking by effects of strong tau pathology in aging P301S mice. We also note that PET imaging paradigms give a macroscopic view of neuroinflammation components in mouse brain as compared to immunohistochemistry or in situ hybridization methods. However, we emphasize the translational relevance of neuroinflammation PET biomarkers [6]. Thus, the synthesis of our combined findings comprise strong evidence for a sex-specific effect on the TSPO-PET signal in aging wild-type mice, and the association between microglia activation with $A \beta$ accumulation in transgenic mice, without corresponding association with tauopathy in P301S mice. Present observations are hypothesis generating for studies of microglial activation by manipulation of sex hormones, i.e., by ovariectomy or orchidectomy [10]. Furthermore, cross-breeding of $A \beta$ and tauopathy mouse models with TSPO knock-out mice [38] could mechanistically help elucidate the effect of sex differences of TSPO on functionally related pathways such as cholesterol synthesis. We note the present imbalance of numbers of male and female mice, which arises from retrospective design of this study.

There is growing evidence that tau pathology follows accumulation of $\mathrm{A} \beta$ in $\mathrm{AD}$ patients [39]. Recent data showed that NLRP3 inflammasome activation triggers tau pathology [40], and importantly, there is evidence that women present a higher level of tau pathology even at the preclinical stage of $\mathrm{AD}$ [41]. This stands in contrast to present finding of absent sex differences in tauopathy in the P301S model mice, which lack $A \beta$ pathology. Thus, enhanced microglial activation in response to $A \beta$ could constitute the key factor resulting 
elevated in tau pathology in female AD patients. TSPOPET in conjunction with tau- and/or A $\beta$-PET in cohorts of $\mathrm{AD}$ and primary tauopathy patients (i.e., progressive supranuclear palsy or corticobasal degeneration) would translate our present findings into the study of human disease.

\section{Conclusions}

Sex-specific effects on the age-dependent increase in cortical TSPO-PET signal are present in wild-type mice and in mouse models of neurodegenerative diseases. Sex moderates the cortical microgliosis in response to amyloidosis but not to tau pathology in transgenic mice. Strict controlling for sex is required for TSPO-PET studies of microglial activation in mouse models of neurodegenerative diseases and requires attention in corresponding studies in human disease.

\section{Abbreviations}

Aß: Beta amyloid; AD: Alzheimer's disease; ANOVA: Analysis of variance; APP: Amyloid precursor protein; B/SE: Main and interaction effects; IHC: Immunohistochemistry; MRI: Magnetic resonance imaging; PET: Positron emission tomography; M: Months; MAPT: Microtubule-associated protein tau; MCl: Mild cognitive impairment; SPM: Statistical parametric mapping; SUV: Standardized uptake value; SUVR: SUV ratio; TSPO: Translocator protein VOls: Volumes of interest; WT: Wild-type

\section{Acknowledgements}

We thank Karin Bormann-Giglmaier and Diana Mahlstedt for excellent technical assistance. App ${ }^{N L-G-F}$ mice were provided by RIKEN BRC through the National Bio-Resource Project of the MEXT, Japan. GE Healthcare manufactured the ${ }^{18} \mathrm{~F}-\mathrm{GE}-180$ cassettes, which were made available through an early-access agreement. Florbetaben precursor was kindly provided by Life Molecular Imaging.

\section{Authors' contributions}

$\mathrm{GB}, \mathrm{FE}, \mathrm{CS}$, and FR performed the majority of PET experiments. NF and ME performed the linear mixed models analysis. TB and JML performed immunohistochemistry staining. GB, FE, CS, LB, FR, and $M B$ analyzed and quantified the data. $P B, A R$, and $M B$ performed interpretation of the PET data. SL and FG performed and improved radiochemistry. GB rendered expert IT support. NF, ME, PB, AR, GUH, JH, and MB contributed to the conception and design of the study and interpreted the combined findings. $\mathrm{GB}, \mathrm{MB}$, and PC wrote the manuscript. BU supervised the study as a veterinarian. All authors participated in the generation of the original data, added important intellectual content to the manuscript and provided critical assessment of the current manuscript. All authors read and approved the final manuscript.

\section{Funding}

The study was financially supported by the SyNergy Cluster (J.H., P.B., C.H., and $A . R$ ) and by the Deutsche Forschungsgemeinschaft (DFG) by a dedicated PET imaging grant to M.B. and A.R. (BR4580/1-1 \& RO5194/1-1). G.H. is supported by the German Federal Ministry of Education and Research (01EK1605A HitTau), the NOMIS foundation (FTLD project). Open Access funding enabled and organized by Projekt DEAL.

\section{Availability of data and materials}

The datasets used and/or analyzed during the current study are available from the corresponding author upon reasonable request.

\section{Ethics approval and consent to participate}

All experiments were carried out in compliance with the National Guidelines for Animal Protection, Germany, and following the guidelines of the EU Directive 2010/63/EU. Experiments were approved by the regional Animal
Care Committee of the Government of Oberbayern (Regierung Oberbayern) and were overseen by a veterinarian.

\section{Consent for publication}

Not applicable.

\section{Competing interests}

G.U.H. received research support from GE Healthcare and Neuropore; has ongoing research collaborations with Orion and Prothena; serves as a consultant for AbbVie, AlzProtect, Asceneuron, Biogen, Biohaven, Lundbeck, Novartis, Roche, Sanofi, and UCB; received honoraria for scientific presentations from AbbVie, Biogen, Roche, Teva, UCB, and Zambon; and holds a patent on PERK Activation for the Treatment of Neurodegenerative Diseases (PCT/EP2015/068734). M.B. received speaking honoraria from Life Molecular Imaging and GE healthcare. M.B. is an advisor of Life Molecular Imaging. All other authors report no conflicts of interest.

\section{Author details}

${ }^{1}$ Department of Nuclear Medicine, University Hospital of Munich, LMU Munich, Marchioninstraße 15, 81377 Munich, Germany. ${ }^{2}$ Institute for Stroke and Dementia Research, University Hospital of Munich, LMU Munich, Munich, Germany. ${ }^{3}$ DZNE - German Center for Neurodegenerative Diseases, Munich, Germany. ${ }^{4}$ Department of Nuclear Medicine, Inselspital, University Hospital Bern, Bern, Switzerland. ${ }^{5}$ School of Psychology and Counselling, Queensland University of Technology, Brisbane, Australia. ${ }^{6}$ Munich Cluster for Systems Neurology (SyNergy), Munich, Germany. ${ }^{7}$ Department of Neurology, Hannover Medical School, Hannover, Germany. ${ }^{8}$ Department of Neurology, Technical University Munich, Munich, Germany. ${ }^{9}$ Center of Neuropathology and Prion Research, University of Munich, Munich, Germany.

Received: 20 July 2020 Accepted: 25 November 2020

Published online: 14 December 2020

References

1. Ziegler-Graham K, Brookmeyer R, Johnson E, Arrighi HM. Worldwide variation in the doubling time of Alzheimer's disease incidence rates. Alzheimers Dement. 2008;4:316-23.

2. Braak H, Braak E. Demonstration of amyloid deposits and neurofibrillary changes in whole brain sections. Brain Pathol. 1991;1:213-6.

3. Hyman BT, Phelps CH, Beach TG, Bigio EH, Cairns NJ, Carrillo MC, Dickson DW, Duyckaerts C, Frosch MP, Masliah E, et al. National Institute on Aging Alzheimer's Association guidelines for the neuropathologic assessment of Alzheimer's disease. Alzheimers Dement. 2012:8:1-13.

4. Serrano-Pozo A, Frosch MP, Masliah E, Hyman BT. Neuropathological alterations in Alzheimer disease. Cold Spring Harb Perspect Med. 2011;1 a006189.

5. $\quad$ Querfurth HW, LaFerla FM. Alzheimer's disease. N Engl J Med. 2010;362:329_ 44.

6. Heneka MT, Carson MJ, Khoury JE, Landreth GE, Brosseron F, Feinstein DL, Jacobs AH, Wyss-Coray T, Vitorica J, Ransohoff RM, et al. Neuroinflammation in Alzheimer's disease. Lancet Neurol. 2015;14:388-405.

7. Stefaniak J, O'Brien J. Imaging of neuroinflammation in dementia: a review. J Neurol Neurosurg Psychiatry. 2016;87:21-8.

8. Cumming P, Burgher B, Patkar O, Breakspear M, Vasdev N, Thomas P, Liu GJ, Banati R. Sifting through the surfeit of neuroinflammation tracers. J Cereb Blood Flow Metab. 2018;38:204-24.

9. Werry EL, Bright FM, Piguet O, Ittner LM, Halliday GM, Hodges JR, Kiernan MC, Loy CT, Kril JJ, Kassiou M. Recent developments in TSPO PET imaging as a biomarker of neuroinflammation in neurodegenerative disorders. Int J Mol Sci. 2019;20:3161.

10. Pike CJ. Sex and the development of Alzheimer's disease. J Neurosci Res. 2017:95:671-80

11. Podcasy JL, Epperson CN. Considering sex and gender in Alzheimer disease and other dementias. Dialogues Clin Neurosci. 2016;18:437-46.

12. Lin KA, Choudhury KR, Rathakrishnan BG, Marks DM, Petrella JR, Doraiswamy PM. Alzheimer's Disease Neuroimaging I: Marked gender differences in progression of mild cognitive impairment over 8 years. Alzheimers Dement (N Y). 2015;1:103-10

13. Kodama L, Gan L. Do microglial sex differences contribute to sex differences in neurodegenerative diseases? Trends Mol Med. 2019;25:741-9. 
14. Santos-Galindo M, Acaz-Fonseca E, Bellini MJ, Garcia-Segura LM. Sex differences in the inflammatory response of primary astrocytes to lipopolysaccharide. Biol Sex Differ. 2011;2:7.

15. Nissen JC. Microglial function across the spectrum of age and gender. Int J Mol Sci. 2017;18:561.

16. Uchoa MF, Moser VA, Pike CJ. Interactions between inflammation, sex steroids, and Alzheimer's disease risk factors. Front Neuroendocrinol. 2016; 43:60-82.

17. Tuisku J, Plaven-Sigray P, Gaiser EC, Airas L, Al-Abdulrasul H, Bruck A, Carson $\mathrm{RE}$, Chen MK, Cosgrove KP, Ekblad L, et al. Effects of age, BMI and sex on the glial cell marker TSPO - a multicentre [(11)C]PBR28 HRRT PET study. Eur J Nucl Med Mol Imaging. 2019;46:2329-38.

18. Garcia-Segura LM, Melcangi RC. Steroids and glial cell function. Glia. 2006;54: 485-98.

19. Brendel M, Probst F, Jaworska A, Overhoff F, Korzhova V, Albert NL, Beck R Lindner S, Gildehaus FJ, Baumann K, et al. Glial activation and glucose metabolism in a transgenic amyloid mouse model: a triple-tracer PET study. J Nucl Med. 2016;57:954-60.

20. Parhizkar S, Arzberger T, Brendel M, Kleinberger G, Deussing M, Focke C, Nuscher B, Xiong M, Ghasemigharagoz A, Katzmarski N, et al. Loss of TREM2 function increases amyloid seeding but reduces plaque-associated ApoE. Nat Neurosci. 2019;22:191-204.

21. Eckenweber F, Medina-Luque J, Blume T, Sacher C, Biechele G, Wind K, Deussing M, Briel N, Lindner S, Boening G, et al. Longitudinal TSPO expression in tau transgenic P301S mice predicts increased tau accumulation and deteriorated spatial learning. J Neuroinflammation. 2020;17:208.

22. Blume T, Focke C, Peters F, Deussing M, Albert NL, Lindner S, Gildehaus FJ, von Ungern-Sternberg B, Ozmen L, Baumann K, et al. Microglial response to increasing amyloid load saturates with aging: a longitudinal dual tracer in vivo muPET-study. J Neuroinflammation. 2018;15:307.

23. Masuda A, Kobayashi Y, Kogo N, Saito T, Saido TC, Itohara S. Cognitive deficits in single App knock-in mouse models. Neurobiol Learn Mem. 2016; 135:73-82.

24. Saito T, Matsuba Y, Mihira N, Takano J, Nilsson P, Itohara S, Iwata N, Saido TC. Single App knock-in mouse models of Alzheimer's disease. Nat Neurosci. 2014;17:661-3.

25. Sacher C, Blume T, Beyer L, Peters F, Eckenweber F, Sgobio C, Deussing M, Albert $\mathrm{NL}$, Unterrainer $\mathrm{M}$, Lindner $\mathrm{S}$, et al. Longitudinal PET monitoring of amyloidosis and microglial activation in a second-generation amyloid-beta mouse model. J Nucl Med. 2019;60:1787-93.

26. Sacher C, Blume T, Beyer L, Biechele G, Sauerbeck J, Eckenweber F, Deussing M, Focke C, Parhizkar S, Lindner S, et al. Asymmetry of fibrillar plaque burden in amyloid mouse models. J Nucl Med. 2020;61:1825-31.

27. Allen B, Ingram E, Takao M, Smith MJ, Jakes R, Virdee K, Yoshida H, Holzer M, Craxton M, Emson PC, et al. Abundant tau filaments and nonapoptotic neurodegeneration in transgenic mice expressing human P301S tau protein J Neurosci. 2002;22:9340-51.

28. Xu H, Rosler TW, Carlsson T, de Andrade A, Bruch J, Hollerhage M, Oertel WH, Hoglinger GU. Memory deficits correlate with tau and spine pathology in P301S MAPT transgenic mice. Neuropathol Appl Neurobiol. 2014;40:833-43.

29. Overhoff F, Brendel M, Jaworska A, Korzhova V, Delker A, Probst F, Focke C, Gildehaus FJ, Carlsen J, Baumann K, et al. Automated spatial brain normalization and hindbrain white matter reference tissue give improved [(18)F]-florbetaben PET quantitation in Alzheimer's model mice. Front Neurosci. 2016:10:45

30. Brendel M, Focke C, Blume T, Peters F, Deussing M, Probst F, Jaworska A, Overhoff F, Albert N, Lindner S, et al. Time courses of cortical glucose metabolism and microglial activity across the life span of wild-type mice: a PET study. J Nucl Med. 2017;58:1984-90.

31. Flurkey K, Currer JM, Harrison D. Mouse models in aging research. In The mouse in biomedical research: Elsevier; 2007;3:637-72.

32. Koscik RL, Betthauser TJ, Jonaitis EM, Allison SL, Clark LR, Hermann BP, Cody KA, Engle JW, Barnhart TE, Stone CK, et al. Amyloid duration is associated with preclinical cognitive decline and tau PET. Alzheimers Dement (Amst). 2020;12:e12007.

33. Jack CR Jr, Bennett DA, Blennow K, Carrillo MC, Dunn B, Haeberlein SB, Holtzman DM, Jagust W, Jessen F, Karlawish J, et al. NIA-AA research framework: toward a biological definition of Alzheimer's disease. Alzheimers Dement. 2018;14:535-62.
34. Carroll JC, Rosario ER, Kreimer S, Villamagna A, Gentzschein E, Stanczyk FZ, Pike CJ. Sex differences in $\beta$-amyloid accumulation in 3xTg-AD mice: role of neonatal sex steroid hormone exposure. Brain research. 2010;1366:233-45.

35. Monasor LS, Müller SA, Colombo A, König J, Roth S, Liesz A, Berghofer A, Saito T, Saido TC, Herms J, et al. Fibrillar A $\beta$ triggers microglial proteome alterations and dysfunction in Alzheimer mouse models. eLife. 2020;9: e54083.

36. Pannell M, Economopoulos $V$, Wilson TC, Kersemans V, Isenegger PG, Larkin JR, Smart S, Gilchrist S, Gouverneur V, Sibson NR. Imaging of translocator protein upregulation is selective for pro-inflammatory polarized astrocytes and microglia. Glia. 2020;68:280-97.

37. Kodama L, Guzman E, Etchegaray Jl, Li Y, Sayed FA, Zhou L, Zhou Y, Zhan L, Le D, Udeochu JC, et al. Microglial microRNAs mediate sex-specific responses to tau pathology. Nat Neurosci. 2020;23:167-71.

38. Barron AM, Ji B, Kito S, Suhara T, Higuchi M. Steroidogenic abnormalities in translocator protein knockout mice and significance in the aging male. Biochem J. 2018;475:75-85.

39. Bloom GS. Amyloid-beta and tau: the trigger and bullet in Alzheimer disease pathogenesis. JAMA Neurol. 2014;71:505-8.

40. Ising C, Venegas C, Zhang S, Scheiblich H, Schmidt SV, Vieira-Saecker A, Schwartz S, Albasset S, McManus RM, Tejera D, et al. NLRP3 inflammasome activation drives tau pathology. Nature. 2019:575:669-73.

41. Buckley RF, Mormino EC, Rabin JS, Hohman TJ, Landau S, Hanseeuw BJ, Jacobs HIL, Papp KV, Amariglio RE, Properzi MJ, et al. Sex differences in the association of global amyloid and regional tau deposition measured by positron emission tomography in clinically normal older adults. JAMA Neurol. 2019;76:542-51.

\section{Publisher's Note}

Springer Nature remains neutral with regard to jurisdictional claims in published maps and institutional affiliations.
Ready to submit your research? Choose BMC and benefit from:

- fast, convenient online submission

- thorough peer review by experienced researchers in your field

- rapid publication on acceptance

- support for research data, including large and complex data types

- gold Open Access which fosters wider collaboration and increased citations

- maximum visibility for your research: over $100 \mathrm{M}$ website views per year

At $\mathrm{BMC}$, research is always in progress.

Learn more biomedcentral.com/submissions 